\title{
Anaphylaxis to Bovine Serum Albumin Tissue Adhesive in a Non-Meat-Allergic Patient
}

Hilger $\mathrm{C}^{1}$, Clark $\mathrm{E}^{2}$, Swiontek $\mathrm{K}^{1}$, Chiriac $\mathrm{AM}^{3,4}$, Caimmi DP ${ }^{3,4}$, Demoly $\mathrm{P}^{3,4,5}$, Bourrain $\mathrm{JL}^{2,3}$

${ }^{1}$ Department of Infection and Immunity, Luxembourg Institute of Health, Esch-sur-Alzette, Luxembourg

${ }^{2}$ Department of Dermatology, CHU Montpellier, University of Montpellier I, Montpellier, France

${ }^{3}$ Department of Pulmonology, Division of Allergy, Hôpital Arnaud de Villeneuve, University, Hospital of Montpellier, Montpellier, France

${ }^{4}$ Sorbonne Université, INSERM UMR-S 1136, IPLESP, Equipe EPAR, Paris, France

${ }^{5}$ WHO Collaborating Centre on Scientific Classification Support, Montpellier, France

J Investig Allergol Clin Immunol 2020; Vol. 30(5): 369-371 doi: $10.18176 /$ jiaci.0522

Key words: BioGlue. Bovine serum albumin. Perioperative anaphylaxis. Pork-cat syndrome.

Palabras clave: BioGlue. Albúmina de suero bovino. Anafilaxia perioperative. Síndrome cerdo-gato.

BioGlue (Cryolife) is a surgical adhesive that has been used since 1998 as an adjunct to standard methods of achieving hemostasis in adult patients during open surgical repair of large vessels. The adhesive is therefore commonly used as surgical sealant in thoracic surgery [1]. It is composed of purified bovine serum albumin (BSA) and glutaraldehyde, which are dispensed by means of a controlled delivery system. Upon mixing, the components polymerize into a flexible mechanical seal. The contraindications are limited (sensitivity to materials of bovine origin, intravascular use, cerebrovascular repair). Since its introduction, application of BioGlue has been expanded notably to include repair of traumatic liver laceration [2].

A 67-year-old atopic man was admitted to hospital for treatment of an aortic aneurysm. A Bentall procedure was performed using BioGlue. The patient experienced anaphylactic shock with bronchospasm and hypotension shortly after application and was treated with crystalloids, norepinephrine, epinephrine, and salbutamol. A blood sample taken during the event showed elevated serum tryptase $(18.5 \mu \mathrm{g} / \mathrm{L})$. The patient was referred to our allergology department for evaluation. He had a history of allergic rhinitis to cat dander, but no food allergies. The results of skin prick tests with cat, dog, hamster, guinea pig, and rabbit dander were positive. Specific IgE (sIgE) was positive for Fel d 1 $\left(21.6 \mathrm{kU}_{\mathrm{A}} / \mathrm{L}\right)$, Fel d $2\left(3.7 \mathrm{kU}_{\mathrm{A}} / \mathrm{L}\right)$, and Fel d $4\left(10.7 \mathrm{kU}_{\mathrm{A}} / \mathrm{L}\right)$ (ImmunoCAP, Thermo Fisher Scientific), but negative for Bos d 6. Skin prick tests were performed using both components of BioGlue, ie, native BSA and glutaraldehyde. The test was positive for BSA $(5 \mathrm{~mm})$ and negative for glutaraldehyde and the mixture of both. 
A

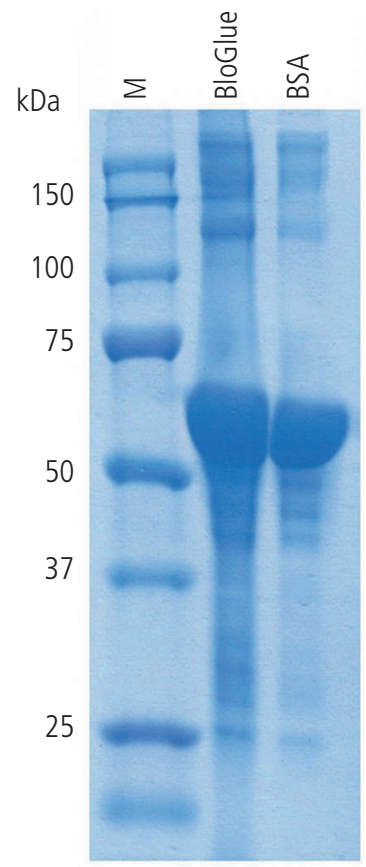

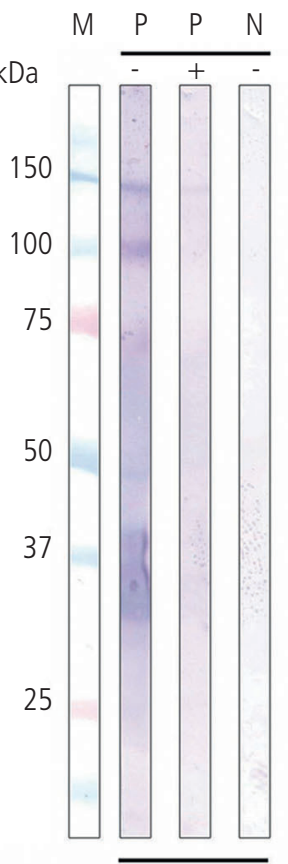

BioGlue
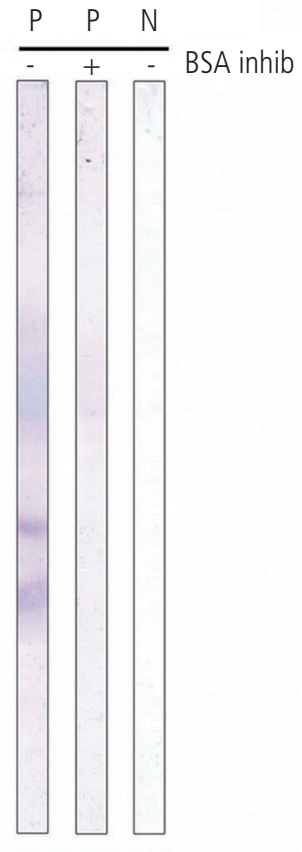

BSA
B

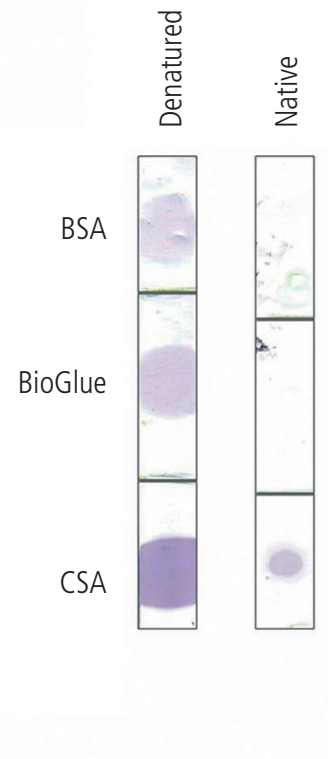

Figure. IgE reactivity to bovine serum albumin (BSA). A, The BSA component of BioGlue (BioGlue) and a commercial BSA preparation were separated in Coomassie blue-stained SDS-PAGE gel (left) and immunoblotted with the patient's serum (right). Cold water fish gelatin ( $2 \%$ ) (Sigma) was used as blocking buffer. IgE reactivity to BioGlue and BSA was completely inhibited by addition of BSA. P indicates patient serum; N, negative control. B, Cat serum albumin (CSA), BioGlue and BSA were spotted onto nitrocellulose under denaturing or native conditions (10 $\mu \mathrm{g} / \mathrm{spot})$ and immunoblotted. IgE reactivity was markedly enhanced under denaturing conditions.

In order to further elucidate the target of the patient's IgE antibodies, we performed immunoblot inhibition assays on the BSA component of BioGlue and commercial BSA (SigmaAldrich). Proteins were separated by Coomassie-stained SDS-PAGE gel or immunoblotted as reported elsewhere [3] (Figure, A). IgE-reactivity was directed mostly toward high $(>100 \mathrm{kDa})$ and low $(35-40 \mathrm{kDa})$ molecular weight components in BioGlue and BSA samples. Reactivity was completely abolished by inhibition with BSA $(100 \mu \mathrm{g} / \mathrm{mL})$, thus confirming that all reactivity was directed toward the BSA component of BioGlue. In order to further understand the discrepancy between a positive immunoblot to BSA and a negative IgE test by ImmunoCAP, we assayed IgE binding to BSA in both the native and the denatured form (Figure, B). BSA was denatured using $1 \%$ SDS and $0.35 \mathrm{M} \beta$-mercaptoethanol and spotted onto a nitrocellulose membrane. IgE-reactivity to denatured cat serum albumin, the BSA component of BioGlue, and BSA is much stronger than that to native albumin, suggesting that $\operatorname{IgE}$ binding is predominantly directed against nonconformational epitopes, thus explaining the negative ImmunoCAP result with Bos d 6. A similar case of anaphylactic shock to BioGlue was reported in a patient who presented with pork-cat syndrome, although the test results for Bos d 6 were negative [4]. The patient in the present case did not report any symptoms upon ingestion of meat but has owned a cat for several years and has a history of allergic rhinitis in the presence of cats. We hypothesize that cross-reactive Fel d 2 epitopes in BSA would be exposed briefly to $\mathrm{IgE}$ antibodies during denaturation when mixing the 2 components, ie, BSA and glutaraldehyde. Solidification of the mixture and strong protein cross-linking would then preclude any further IgE binding and lead to an asymptomatic outcome. The severity of the reaction is probably due to the high concentration of BSA used in the product. A recent report on anaphylaxis to intravenous human serum albumin supports the idea that treated albumin could present structural changes capable of inducing sensitization and subsequent allergic responses upon administration. The patient was tolerant to human blood and plasma derivatives and did not present sIgE to animal serum albumins [5].

The present case shows that patients with sIgE to Fel d 2, even at low levels, may have a high risk of perioperative anaphylaxis upon using BioGlue as tissue adhesive. Catallergic patients should be screened for sIgE to Fel d 2 before undergoing thoracic surgery. Up to $14 \%-23 \%$ of cat-allergic patients have been reported to be sensitized to Fel d 2, and about $1 \%-3 \%$ are likely to present pork-cat syndrome [6]. Despite the fact that surgical sealants composed of purified BSA are widely used, this is only the second case reported in the literature. In addition to sIgE against Fel d 2, other, as yet unidentified cofactors might lead to the severe reactions described in the present case and reported by Dewachter et al [4]. The risk-benefit balance should be carefully assessed for each patient and alternative sealants considered. 
In conclusion, we report an additional case of perioperative anaphylaxis to BioGlue. We confirm the importance of $\mathrm{IgE}$ screening for sensitization to Fel d 2 in cat-allergic patients, although we also wish to caution clinicians against the fact that the finding of a negative $\operatorname{IgE}$ result for Bos $\mathrm{d} 6$ and tolerance of bovine or porcine meat does not exclude a potential risk of reactivity to BSA-based tissue adhesives.

\section{Funding}

The study was supported by institutional funding provided by the Ministry of Higher Education and Research, Luxembourg.

\section{Conflicts of Interest}

The authors declare that they have no conflicts of interest.

\section{References}

1. Tsilimigras DI, Antonopoulou A, Ntanasis-Stathopoulos I, Patrini D, Papagiannopoulos K, Lawrence D, et al. The role of BioGlue in thoracic surgery: a systematic review. J Thorac Dis. 2017;9:568-76.

2. Daniele $E$, Dissanaike $S$. BioGlue for traumatic liver laceration. Int J Surg Case Rep. 2016;23:33-5.

3. Swiontek K, Morisset M, Codreanu-Morel F, Fischer J, Mehlich J, Darsow U, et al. Drugs of porcine origin-A risk for patients with $\alpha$-gal syndrome? J Allergy Clin Immunol Pract. 2019;7:1687-90.e3.

4. Dewachter P, Jacquenet S, Beloucif S, Goarin JP, Koskas F, Mouton-Faivre C. Pork-cat syndrome revealed after surgery: Anaphylaxis to bovine serum albumin tissue adhesive. J Allergy Clin Immunol Pract. 2019;7:2450-2.

5. Moreno Lozano L, Galindo Bonilla P, Borja Segade J, Extremera Ortega A, Gómez Torrijos E, García Rodríguez R. Human Serum Albumin Induced Anaphylaxis in a Patient With Good Tolerance to Human Plasma. J Invest Allergol Clin Immunol. 2019;29:51-3.

6. Hilger C, Kohnen M, Grigioni F, Lehners C, Hentges F. Allergic cross-reactions between cat and pig serum albumin. Study at the protein and DNA levels. Allergy. 1997;52:179-87.

Manuscript received February 5, 2020; accepted for publication March 27, 2020.

Jean Luc Bourrain Allergologie Hôpital Arnaud de Villeneuve 371 Avenue du Doyen Gaston Giraud 34090 Montpellier, France E-mail: Jl-bourrain@chu-montpellier.fr 